\title{
Molecular Mechanisms of COMPLEXIN Fusion Clamp Function in Synaptic Exocytosis Revealed in a New Drosophila Mutant
}

\author{
Janani lyer, Christopher J. Wahlmark, Giselle A. Kuser-Ahnert, and Fumiko Kawasaki \\ Department of Biology and Center for Molecular Investigation of Neurological Disorders Penn \\ State University, University Park, PA 16802
}

\begin{abstract}
The COMPLEXIN (CPX) proteins play a critical role in synaptic vesicle fusion and neurotransmitter release. Previous studies demonstrated that CPX functions in both activation of evoked neurotransmitter release and inhibition/clamping of spontaneous synaptic vesicle fusion. Here we report a new $c p x$ mutant in Drosophila melanogaster, $c p x^{1257}$, revealing spatially defined and separable pools of CPX which make distinct contributions to the activation and clamping functions. In $c p X^{1257}$, lack of only the last C-terminal amino acid of CPX is predicted to disrupt prenylation and membrane targeting of CPX. Immunocytochemical analysis established localization of wild-type CPX to active zone (AZ) regions containing neurotransmitter release sites as well as broader presynaptic membrane compartments including synaptic vesicles. Parallel biochemical studies confirmed CPX membrane association and demonstrated robust binding interactions of CPX with all three SNAREs. This is in contrast to the $c p x^{1257}$ mutant, in which AZ localization of CPX persists but general membrane localization and, surprisingly, the bulk of CPX-SNARE protein interactions are abolished. Furthermore, electrophysiological analysis of neuromuscular synapses revealed interesting differences between $c p x^{1257}$ and a $c p x$ null mutant. The $c p x$ null exhibited a marked decrease in the EPSC amplitude, slowed EPSC rise and decay times and an increased mEPSC frequency with respect to wild-type. In contrast, $c p x^{1257}$ exhibited a wild-type EPSC with an increased mEPSC frequency and thus a selective failure to clamp spontaneous release. These results indicate that spatially distinct and separable interactions of CPX with presynaptic membranes and SNARE proteins mediate separable activation and clamping functions of CPX in neurotransmitter release.
\end{abstract}

\section{Keywords}

Neurotransmitter release; Farnesylation; Neuromuscular; SNARE; DLM

\section{INTRODUCTION}

It is widely accepted that SNARE proteins function at the core of the neurotransmitter release apparatus, where they promote exocytotic fusion of neurotransmitter-filled synaptic

(C) 2013 Elsevier Inc. All rights reserved

Corresponding Author: Fumiko Kawasaki Department of Biology and Center for Molecular Investigation of Neurological Disorders 201 Life Sciences Building, Penn State University, University Park, PA 16802 Phone: 814-865-6863, Fax: 814-865-7280, fxk6@psu.edu.

Publisher's Disclaimer: This is a PDF file of an unedited manuscript that has been accepted for publication. As a service to our customers we are providing this early version of the manuscript. The manuscript will undergo copyediting, typesetting, and review of the resulting proof before it is published in its final citable form. Please note that during the production process errors may be discovered which could affect the content, and all legal disclaimers that apply to the journal pertain. 
vesicles with the presynaptic plasma membrane (Jahn and Scheller, 2006). However, defining the mechanisms which provide precise and rapid regulation of synaptic vesicle fusion remains among the foremost problems in cellular and molecular neuroscience. The identification of CPX as a protein which binds and regulates SNARE complexes (Ishizuka et al., 1995; McMahon et al., 1995) has advanced our understanding of these mechanisms (Brose, 2008; Neher, 2010; Rizo and Rosenmund, 2008; Stein and Jahn, 2009; Südhof and Rothman, 2009). Notably, CPX can both promote SV fusion evoked by a presynaptic action potential and suppress or "clamp" spontaneous vesicle fusion. Recent models suggest that specific domains of CPX (Fig. 1A) contribute to different aspects of synaptic vesicle fusion (Hobson et al., 2011; Martin et al., 2011; Maximov et al., 2009; Reim et al., 2001; Strenzke et al., 2009; Tang et al., 2006; Xue et al., 2010; Xue et al., 2009; Xue et al., 2007; Yang et al., 2010). Whereas a "central helix" which binds SNARE complexes (Bracher et al., 2002; Chen et al., 2002) is absolutely required for CPX function, other domains appear to mediate specific aspects of CPX activity (Rizo and Rosenmund, 2008; Stein and Jahn, 2009). For example, recent studies have shown that the CPX C-terminus is specifically required for the clamping function (Buhl et al., 2013; Cho et al., 2010; Kaeser-Woo et al., 2012; Martin et al., 2011; Xue et al., 2009). Of particular relevance to the present study is a specific CaaX motif found at the extreme C-terminus of several mammalian and Drosophila CPX isoforms. This motif has been shown to mediate CPX prenylation [a form of lipid modification; (Omer and Gibbs, 1994; Resh, 2006)] and has been implicated in both targeting CPX to membranes (Reim et al., 2005) and the CPX clamping function (Cho et al., 2010; Xue et al., 2009). The form of prenylation demonstrated for mammalian CPX isoforms (CPX3 and 4) is farnesylation (Reim et al., 2005), consistent with previous studies indicating that one of several specific residues in the X position of the CaaX motif (A,C,M,Q,S) selectively mediates farnesylation (Omer and Gibbs, 1994).

This progress is extended by new insights gained from the present study, in which the isolation and characterization of a new $c p x$ mutant further defines the in vivo molecular basis of CPX functions and interactions within the neurotransmitter release apparatus. This study reveals a specific subcellular distribution for CPX within the presynaptic terminal and a role for C-terminal farnesylation in mediating both association of CPX with presynaptic membranes and CPX clamping of spontaneous synaptic vesicle fusion.

\section{MATERIALS AND METHODS}

\section{Drosophila Strains}

$A p p l-G A L 4$ and $w ; ; L y / T M 6 c$ were from our laboratory stock collection. The $c p x^{S H 1}$ null mutant and the $U A S$-cpx transgenic line were generously provided by Troy Littleton (MIT, Cambridge, MA). Deficiency lines, $D f(3 L) G N 34$ and $D f(3 R)$ Exel6140, were obtained from the Bloomington Stock Center. UAS-EGFP-cpx and UAS-EGFP-cpx ${ }^{1257}$ transgenic lines were generated in the current study (see "Generation of Transgenic lines"). Stocks and crosses were cultured on a conventional cornmeal-molasses-yeast medium at $20^{\circ} \mathrm{C}$ for electrophysiological analysis or at room temperature. Wild-type flies were Canton-S.

\section{Mutagenesis and Screening}

A genetic screen was carried out to recover new hypomorphic and conditional alleles of $c p x$. Briefly, Iso3 males carrying an isogenized third chromosome were exposed to $25 \mathrm{mM}$ ethyl methanesulfonate (EMS) for 24 hours (Dellinger et al., 2000). F2 flies heterozygous for a mutagenized third chromosome in trans to the third chromosome carrying deficiencies, $D f(3 L) G N 34$ and $D f(3 R) E x e l 6140$, were screened for motor defects at $38{ }^{\circ} \mathrm{C}$. The latter deficiency removes the $c p x$ locus. 


\section{Molecular Characterization of the $\mathrm{cpx}^{1257}$ Mutant}

Sequence analysis of the $c p x$ open reading frame (ORF) was carried out essentially as described previously (Lutas et al., 2012). Briefly, genomic DNA was prepared from the $c p x$ mutant and used as template for PCR. Gel-purified PCR products were sequenced at the Penn State University Nucleic Acids Facility. Sequences from the $c p x$ mutant were compared to those from the parent third chromosome used in the mutagenesis. The location of the $c p x^{1257}$ mutation within an alternative exon is defined further in the following section.

\section{Relevant information about cpx alternative exons and splice variants}

As described in the Results, the $c p x^{1257}$ mutation was found to be isoform-specific in that it maps to an alternative exon. As indicated in flybase (http://www.flybase.org), the position of this exon within the genomic DNA sequence begins at 3R:123210 and ends at 3R:124307. Splice variants including this exon are exemplified here by transcript $c p x$-RU. The single known splice variant lacking this exon is transcript $c p x$-RE. The $c p x$ coding sequence of the $U A S-E G F P$-cpx and $U A S-E G F P-c p x^{1257}$ transgenes corresponds to that of transcript $c p x-$ $\mathrm{RU}$. The $c p x$ coding sequence of the $U A S$-cpX corresponds to that of transcript $c p x$-RE.

\section{Generation of Transgenic Lines}

Transformation construct for $U A S$-EGFP-cpx was generated by inserting the $c p x$ ORF with EGFP fused to its $\mathrm{N}$-terminus into the $\mathrm{P}$ element transformation vector, pUAST (Brand and Perrimon, 1993). The $c p x$ ORF was amplified from a cDNA clone (clone ID: GH27718; GenBank accession number: AY121629; corresponds to transcript $c p x$-RU) obtained from the Drosophila Genomics Research Center. In the case of UAS-EGFP-cpx ${ }^{1257}$, the codon for the last amino acid of the $c p x$ ORF was removed. Transgenic lines were generated as described previously (Kawasaki et al., 2004). Neural expression of UAS transgenes was achieved using the Appl-GAL4 driver.

\section{Immunocytochemistry and Confocal Microscopy}

Immunocytochemistry and Confocal Microscopy were performed essentially as described previously (Kawasaki et al., 2011; Lutas et al., 2012). These studies employed the following primary antibodies: rabbit polyclonal anti-CPX antibody $(1: 1,000)$ [Troy Littleton (MIT, Cambridge, MA)]; rabbit anti-SYNAPTOTAGMIN (SYT) Dsyt CL1 $(1: 5,000)$ [Noreen Reist (Colorado State University, Fort Collins, CO)]; mAb nc82 anti-BRUCHPILOT (BRP) (1:50) (Developmental Studies Hybridoma Bank, University of Iowa, Iowa City, IA, U.S.A.); Cy5-conjugated rabbit anti-HRP (1:200) (Jackson Immunoresearch Laboratories, West Grove, PA). Secondary antibodies included Alexa Fluor 488-conjugated anti-mouse $\operatorname{IgG}(1: 200)$, Alexa Fluor 568-conjugated anti-mouse IgG (1:200) and Alexa Fluor 568conjugated anti-rabbit IgG (1:200) (Invitrogen, Carlsbad, CA). Adult DLM neuromuscular synapse preparations were imaged using an Olympus FV1000 confocal microscope (Olympus Optical, Tokyo, Japan) with a PlanApo 60× 1.4 numerical aperture oil objective (Olympus Optical) and a z-step size of $0.2 \mu \mathrm{m}$. Images were obtained and processed with Fluoview software (Olympus Optical). All images shown in figures are maximum projections of two consecutive optical z-sections. Image analysis was carried out in ImageJ. For generating intensity profiles, lines were placed perpendicular to the long axis of the axon and centered at the maximum signal intensity for BRP (in comparisons of BRP and CPX) or $\mathrm{CPX}$ (in comparisons of CPX and SYT).

\section{Western Analysis}

Western analysis of head homogenates was performed using conventional methods as described previously (Kawasaki et al., 2004; Zou et al., 2008). To examine CPX, the equivalent of 0.5 fly heads was loaded per lane on a $12 \%$ SDS-PAGE gel. The primary 
antibody, a rabbit polyclonal anti-CPX antibody (Huntwork and Littleton, 2007) was used at a dilution of 1:10,000. Detection was performed with HRP-conjugated secondary antibodies (Amersham Biosciences, Arlington Heights, IL) and enhanced chemiluminescence (ECL Plus Western Blotting Detection System: Amersham Biosciences, Arlington Heights, IL). TUBULIN served as a loading control and was detected using a monoclonal anti-acetylated a-TUBULIN antibody (Sigma, St. Louis, MO) at a dilution of 1:2,000,000. Analysis following Triton X-114 phase partitioning and co-Immunoprecipitation used the following primary antibodies: mAb JL-8 anti-GFP (1:500) (Clontech, Mountain View, CA), mAb JLA20 anti-ACTIN (1:10) (Developmental Studies Hybridoma Bank), mAb 8C3 antiSYNTAXIN (SYX) (1:10) (Developmental Studies Hybridoma Bank), rabbit anti-SNAP25 antibody $(1: 2,000)$ [David Deitcher (Cornell University, Ithaca, NY)], rabbit anti-NSYB antibody (1:30), [Richard Ordway (Penn State University, State College, PA)]

\section{Triton X-114 phase partitioning}

Triton X-114 extractions were performed essentially as described previously (Bordier, 1981; Mathias et al., 2011; Wang and Coppel, 2002). Precondensation of Triton X-114 (100\%, Sigma) was performed as described to remove the more hydrophilic components from the reagent (Wang and Coppel, 2002). The resulting detergent-enriched phase containing $10 \%$ detergent in PBS (171 mM NaCl, $4 \mathrm{mM} \mathrm{Na}_{2} \mathrm{HPO}_{4}, 3.4 \mathrm{mM} \mathrm{KCl}$ and $1.84 \mathrm{mM} \mathrm{KH}_{2} \mathrm{PO}_{4}, \mathrm{pH}$ 7.5) was used as a stock solution to prepare all other detergent solutions by dilution in PBS. Head homogenates from flies expressing EGFP-CPX, EGFP-CPX ${ }^{1257}$ or soluble EGFP alone as well as Canton-S and $c p x^{1257}$ were prepared by homogenizing 50 heads in $200 \mu \mathrm{l}$ of $1 \%$ Triton X-114 containing a 1:100 protease inhibitor cocktail (Sigma). Following $1 \mathrm{hr}$ incubation on ice and centrifugation for $10 \mathrm{~min}$ at $10,000 \mathrm{~g}$ at $4{ }^{\circ} \mathrm{C}, 150 \mu \mathrm{l}$ of the final head lysate (supernatant) was subjected to phase partitioning. Phase partitioning was achieved by incubating the supernatants at $37^{\circ} \mathrm{C}$ for $15 \mathrm{~min}$, followed by centrifugation at $5000 \mathrm{~g}$ for 15 min at room temperature. The upper aqueous phase was collected and the stock Triton $\mathrm{X}-114$ solution was added to achieve a final volume of $200 \mu \mathrm{l}$ containing a detergent concentration of $\sim 1 \%$. The detergent phase, an oily droplet at the bottom of the tube, was resuspended in $175 \mu \mathrm{l}$ of $0.05 \%$ TritonX-114 to achieve a final volume of $200 \mu \mathrm{l}$ containing a detergent concentration of $\sim 1 \%$. These aqueous and detergent samples were further purified by repeating the phase partitioning process, essentially as described above, three times. After the final partitioning, the proteins were precipitated by adding $100 \%$ TCA to a final concentration of $15 \%$ and incubated on ice for $30 \mathrm{~min}$. Then the pellet was recovered by centrifuging at $10,000 \mathrm{~g}$ for $20 \mathrm{~min}$ at $4{ }^{\circ} \mathrm{C}$, washed in ice-cold acetone at $10,000 \mathrm{~g}$ for 10 min at $4{ }^{\circ} \mathrm{C}$ and air dried. Both the aqueous and the detergent pellets were resuspended in 50 $\mu \mathrm{l}$ of SDS sample buffer [62.5 mM Tris.Cl/SDS, pH 6.8, 2\% SDS, $1 \%$ (v/v) 2mercaptoethanol, $10 \%$ (v/v) glycerol and $0.5 \%$ Bromophenol Blue] and boiled for $3 \mathrm{~min}$. For Western blot analysis, $10 \mu \mathrm{l}$ of the final samples was loaded to a $12 \%$ SDS-PAGE gel.

\section{Co-Immunoprecipitation (Co-IP) analysis}

Co-Immunoprecipitation analysis was carried out essentially as described previously (Yu et al., 2011; Zou et al., 2008). Fly head lysate was prepared from transgenic flies expressing EGFP-CPX or EGFP-CPX ${ }^{1257}$ as well as Canton $S$ controls. Five hundred microliters of lysate equivalent to 300 heads and $5 \mu \mathrm{g}$ of a rabbit polyclonal anti-GFP antibody (Invitrogen, Carlsbad, CA) were incubated with $50 \mu \mathrm{l}$ of a $50 \%$ slurry of ProteinASepharose beads (Amersham Biosciences). After washing, beads were pelleted, resuspended in $40 \mu \mathrm{l}$ of SDS sample buffer and boiled for 3 min to elute proteins. For detection of EGFPCPX or EGFP-CPX ${ }^{1257}$ (IP), the equivalent of 0.5 fly heads for pre-IP input and $4 \mu \mathrm{l}(\sim 30$ heads) of the IP sample were loaded to a $12 \%$ SDS-PAGE gel. To examine co-IP of the SNARE proteins, the equivalent of 0.5 fly heads for pre-IP input and $12 \mu \mathrm{l}$ ( $\sim 90$ heads) of 
the IP sample were loaded into a 12\% SDS-PAGE gel. After electrophoresis, gels were processed for Western analysis.

\section{Electrophysiology}

Two-electrode voltage-clamp recordings of EPSCs and mEPSCs were obtained at $20{ }^{\circ} \mathrm{C}$ from dorsal longitudinal flight muscle (DLM) neuromuscular synapses of 3 to 5 day-old adults and larval neuromuscular synapses of ventral longitudinal muscle 6 in abdominal segment A3. Saline solution consisted of (in $\mathrm{mM}$ ): $129 \mathrm{NaCl}, 2 \mathrm{KCl}, 4.0 \mathrm{MgCl}_{2}, 1.8 \mathrm{CaCl}_{2}$, 5 HEPES, and 36 sucrose. The $\mathrm{pH}$ was adjusted to 7.0 using $\mathrm{NaOH}$. These experiments were performed as described previously (Kawasaki and Ordway, 2009; Wu et al., 2005) with the following exceptions. mEPSC and mEPSP recordings were performed using an AxoClamp-2B amplifier (Axon Instruments, Foster City, CA) and, in the case of recordings at DLM neuromuscular synapses, the membrane potential was held at $-80 \mathrm{mV}$ to improve the signal to noise ratio. Analysis of EPSCs, mEPSCs and mEPSPs was carried out in Mini Analysis (Synaptosoft Inc., Fort Lee, NJ) and IGOR Pro. (WaveMetrics, Inc., Portland, OR).

\section{Data Analysis}

Microsoft (Seattle, WA) Excel was utilized to analyze numerical data and generate graphs. All data values are presented as mean \pm SEM. Statistical significance was determined using the two-tailed Student's $t$ test and significance was assigned to comparisons for which $p \leq$ 0.05 .

\section{RESULTS}

\section{Genetic and Molecular Characterization of a New cpx Mutant}

Further genetic analysis to examine the in vivo molecular mechanisms of CPX function was pursued through a forward genetic screen for new mutant alleles of the single Drosophilacpx gene. To complement a previously reported $c p x$ null mutant (Huntwork and Littleton, 2007), this screen was intended to recover hypomorphic and conditional alleles that may further define the in vivo molecular determinants of CPX function. A screen was performed using chemical mutagenesis and subsequent screening for $c p x$ mutants in F2 progeny carrying a mutagenized third chromosome in trans to a deficiency (deletion) which removes $c p x$ (see Methods). The screen consisted of examining motor behavior at the elevated temperature to detect hypomorphic or temperature-sensitive (TS) phenotypes. One new mutant, initially referred to 1257 , was recovered on the basis of its severe lack of motor co-ordination at 38 ${ }^{\circ} \mathrm{C}$.

On the basis of genetic complementation testing with the $c p x$ null mutant, 1257 was confirmed to be a new allele of $c p x$ and named $c p x^{1257}$. Sequence analysis of the $c p x$ ORF revealed the molecular lesion in $c p x^{1257}$. Remarkably, this mutation removes only the last $\mathrm{C}$-terminal amino acid of CPX, which is a $\mathrm{Q}$ in the $\mathrm{X}$ position of the $\mathrm{C}$-terminal CaaX sequence (Fig. 1A). This residue is required for farnesylation of certain mammalian CPX isoforms (CPX3 and 4) and has been implicated in their membrane targeting (Reim et al., 2005). Western analysis of the $c p x^{1257}$ mutant (Fig. 1B) demonstrated wild-type CPX protein levels in $c p x^{1257}$ [comparing the hemizygous conditions, $c p x^{1257 / D f(3 R) E x e l 6140}$ and $+/ D f(3 R) E x e 16140]$. Thus the $c p x^{1257}$ phenotype appears to reflect the properties of the mutant protein rather than its expression level. Note that $\mathrm{CPX}^{1257}$ migrates at a slightly higher relative molecular mass in comparison to wild-type CPX (Fig. 1B), most likely because of altered post-translational processing. Finally, the $c p x^{1257}$ mutation is isoformspecific in that it occurs in an alternative exon which is present in most isoforms expressed from the single Drosophilacpx gene [(Buhl et al., 2013) and see Fig. 2A and Materials and Methods]. Incidentally, the altered migration of $\mathrm{CPX}^{1257}$ in Western analysis also permits 
the conclusion that CPX isoforms containing this alternative exon, and thus those affected by the $c p x^{1257}$ mutation, are predominant in the nervous system (Fig. 2B). Lastly, final confirmation that $c p x^{1257}$ is an allele of $c p x$ is provided by transformation rescue experiments described in the following section.

\section{Electrophysiological Analysis Reveals a Distinct Synaptic Phenotype in cpx ${ }^{1257}$}

To examine the impact of $c p x^{1257}$ on synaptic function, voltage-clamp analysis of synaptic currents was carried out at adult Dorsal Longitudinal Muscle (DLM) neuromuscular synapses as described previously (Kawasaki and Ordway, 2009). This glutamateric synapse exhibits detailed functional properties similar to those of mammalian synapses (Kawasaki and Ordway, 2009) and has been fruitful in molecular analysis of synaptic function. In part as a basis for comparison, our electrophysiological studies of $c p x$ began with the $c p x{ }^{S H 1}$ null mutant (Huntwork and Littleton, 2007).

Recordings from DLM neuromuscular synapses of the $c p{ }^{S H 1}$ null mutant revealed a drastic reduction in evoked neurotransmitter release, consistent with previous studies of mouse (Reim et al., 2001) and Drosophila (Huntwork and Littleton, 2007; Jorquera et al., 2012) null mutants. $c p x^{S H 1}$ exhibited a $77 \%$ decrease in the peak Excitatory Postsynaptic Current (EPSC) amplitude with respect to wild type (Fig. 3A,B,E). Also as anticipated from previous work, a marked increase in the frequency of miniature EPSCs (mEPSCs) was observed in $c p X^{S H 1}$ (Fig. 3H,I), consistent with loss of CPX function in clamping spontaneous synaptic vesicle fusion. $c p x{ }^{S H 1}$ also exhibited an altered EPSC waveform, as reported previously at larval neuromuscular synapses (Jorquera et al., 2012), which was characterized by a slowing of the EPSC rise and decay times with respect to wild type (Fig. 3C,F,G). These synaptic phenotypes are rescued by neuronal (presynaptic) expression of a wild-type CPX (Fig. 3D$\mathrm{G})$, indicating that each reflects loss of presynaptic CPX function.

With regard to the $c p x^{1257}$, electrophysiological analysis at DLM neuromuscular synapses revealed a synaptic phenotype which was distinct from the $c p x^{S H 1}$ null mutant. Notably, $c p x^{1257}$ exhibits a selective increase in mEPSC frequency (Fig. 3P-R) with no effect on either the amplitude or waveform of the EPSC (Fig. 3J-O). The increased mini frequency in $c p x^{1257}$ was comparable to that observed in the null mutant. Respective mEPSP frequencies in wild type, $c p x^{S H 1}$ and $c p x^{1257}$ were $10.8 \pm 4.97 \mathrm{~Hz}(\mathrm{n}=4), 116.1 \pm 9.16(\mathrm{n}=3)(p=0.00001$ with respect to wild type) and $101.7 \pm 10.55(\mathrm{n}=3)(p=0.00002$ with respect to wild type). These observations indicate that the $c p x^{1257}$ mutation does not alter the roles of CPX in calcium-triggered neurotransmitter release and control of neurotransmitter release kinetics, but selectively disrupts clamping of spontaneous vesicle fusion.

Because previous functional characterization of $c p x$ mutants were carried out at larval neuromuscular synapses, additional studies were carried out for comparison of $c p x$ mutant phenotypes at adult and larval neuromuscular synapses. Voltage clamp analysis of larval neuromuscular synapses in $c p x^{S H 1}$ and $c p x^{1257}$ mutants demonstrated mutant phenotypes similar to those of the adult (Fig. 4). The preceding findings indicate a clear difference in the functional consequences of the $c p x^{1257}$ and $c p x$ null mutations at two different synapses. The molecular basis of this difference with respect to farnesylation of CPX and its clamping function are addressed in the following sections.

\section{The presynaptic distribution of CPX at native synapses: Apparent loss of CPX membrane association in the $\mathrm{cpx}^{1257}$ mutant}

The selective effect of the $c p x^{1257}$ mutation on the clamping function of CPX reflects the underlying molecular lesion, which removes only the last C-terminal amino acid of CPX (the $\mathrm{X}$ in the $\mathrm{C}$-terminal $\mathrm{CaaX}$ motif; Fig. 1). The $\mathrm{CaaX}$ motif is required for farnesylation 
and implicated in membrane targeting of some mammalian CPX isoforms (Reim et al., 2005). Thus we considered that altered protein localization may contribute to the $c p x^{1257}$ synaptic phenotype. This possibility was examined by immunocytochemical studies at DLM neuromuscular synapses. The favorable spatial resolution in this experimental model (Kawasaki and Ordway, 2009) has allowed us to first define the subcellular distribution of CPX within the presynaptic terminal. CPX is highly enriched at the AZ and also detected in a broader pattern which is colocalized with synaptic vesicle markers (Fig. 5A-R). Because synaptic vesicle clusters include a high density of membranes, this distribution of CPX does not exclude a more general presynaptic membrane association, including with the plasma membrane, rather than specific localization to SVs. Several synaptic vesicle proteins which appear to be highly enriched on SVs are also found on the plasma membrane (FernándezAlfonso et al., 2006; Kawasaki and Ordway, 2009; Taubenblatt et al., 1999).

Immunocytochemical studies of $\mathrm{CPX}^{1257}$, which is predicted to lack C-terminal farnesylation, revealed an altered distribution with respect to wild-type CPX. Interestingly, the strong AZ localization observed for wild-type CPX was preserved in this mutant whereas the more diffuse distribution associated with synaptic vesicles/membranes appeared to be lost (Fig. 5S-G'). Thus, a mutation which preserves AZ localization of CPX and disrupts association of CPX with synaptic vesicles/membranes produced a synaptic phenotype characterized by an increased mini frequency and no effect on the EPSC amplitude or waveform.

\section{CPX Membrane Association is lost in the CPX ${ }^{1257}$ Mutant Lacking the CaaX Farnesylation Motif}

Although the presence of a C-terminal farnesylation motif in Drosophila CPX suggests that farnesylation is conserved with respect to mammalian forms, this has not been demonstrated. Given the established role of farnesylation in promoting protein association with membranes, and the preceding immunocytochemical analysis indicating that membrane-targeting of CPX is disrupted in the $c p x^{1257}$ mutant, we anticipated that biochemical studies of $c p x^{1257}$ may reveal differences in CPX association with membranes and possibly its protein binding partners. Previous work demonstrated membrane association of prenylated proteins by partitioning tissue homogenates into aqueous and detergent phases containing cytosolic and membrane-associated proteins, respectively (Price et al., 2010; Reinicke et al., 2005). In the present study, head homogenates from flies expressing EGFPCPX, EGFP-CPX ${ }^{1257}$ or soluble EGFP alone were prepared with Triton X-114 and subjected to phase separation as described previously (Bordier, 1981; Mathias et al., 2011; Wang and Coppel, 2002). Aqueous and detergent phases were examined by Western analysis for the presence of CPX (Fig. 6 A,B). While soluble EGFP partitioned into the aqueous phase as expected, EGFP-CPX was found exclusively in the detergent phase as anticipated for membrane-associated CPX. In contrast, EGFP-CPX ${ }^{1257}$ partitioned solely into the aqueous phase. These results indicate increased membrane association of EGFPCPX with respect to soluble EGFP and that the mechanism mediating membrane association is abolished in EGFP-CPX ${ }^{1257}$ lacking the farnesylation motif. Similar results were obtained for endogenous CPX and CPX ${ }^{1257}$ (Fig. 6 C,D). Together with our immunocytochemical studies, these observations strongly support the conclusion that wild-type CPX associates with presynaptic membrane compartments through its farnesylation. Notably, in the $c p x^{1257}$ mutant, CPX localization at the AZ and its function in evoked synaptic vesicle fusion are preserved and thus $\mathrm{CPX}$ interactions at the $\mathrm{AZ}$ may not require its association with presynaptic membranes. 


\section{Interaction of CPX with SNARE Proteins}

The binding interactions of CPX with ternary SNARE complexes have been well characterized (Bowen et al., 2005; Ishizuka et al., 1995; Li et al., 2007; McMahon et al., 1995; Pabst et al., 2002). To examine whether these interactions are conserved in Drosophila and possibly altered in the $c \mathrm{px}^{1257}$ mutant, co-immunoprecipitation studies of Drosophila CPX and neuronal SNAREs were carried out. Analysis of tissue homogenates was performed using flies expressing EGFP-CPX or EGFP-CPX ${ }^{1257}$ in the nervous system. This is advantageous in that efficient immunoprecipitation (IP) may be achieved using an antiGFP antibody followed by Western analysis of co-imumunoprecipated proteins (Lutas et al., 2012). IP of EGFP-CPX produced robust co-IP of the three endogenous SNARE proteins, SYNTAXIN (SYX), SNAP-25 and neuronal-SYNAPTOBREVIN (NSYB) from fly head homogenates (Fig. 7A,B). The specificity of these interactions was validated by co-IPs from control samples lacking the EGFP-CPX expression (Fig. 7A,B). Similar experiments carried out with homogenates prepared from flies expressing EGFP-CPX ${ }^{1257}$ revealed interesting, and somewhat surprising, results (Fig. 7C,D). For all three SNARE proteins, co-IP with EGFP-CPX ${ }^{1257}$ was drastically reduced with respect to EGFP-CPX.

Given that presynaptic membrane association of CPX was disrupted by the CPX ${ }^{1257}$ mutation (Figs. 5S-G' and 6), these IP results show that most of the observed interactions of wild-type CPX with SNARE proteins involve its association with presynaptic membranes. It is noteworthy that CPX interactions with t-SNAREs, which are localized primarily to the presynaptic plasma membrane [c.f. (Tolar and Pallanck, 1998)], are drastically reduced in the $c p x^{1257}$ mutant despite persistence of CPX localization to the AZ. Taken together, our results indicate that $\mathrm{CPX}$ farnesylation promotes its interaction with the presynaptic plasma membrane, synaptic vesicles and t- and v-SNAREs (see Discussion). Conversely, note that some persistent interactions between $\mathrm{CPX}^{1257}$ and SNAREs were also evident in the co-IPs (Fig. 7D). It is of interest to consider that these may correspond to the preserved CPX ${ }^{1257}$ localization at the AZ (Fig. 5S-X,F', G') where t-SNAREs are localized (Kawasaki and Ordway, 2009) and thought to engage in trans-SNARE complexes.

\section{DISCUSSION}

Through characterization of a new Drosophilacpx mutant, the present study advances our understanding of the molecular mechanisms mediating CPX function in neurotransmitter release. Our findings provide new information about the subcellular distribution of CPX with respect to presynaptic membranes, as well as its molecular basis, and implicate CPX membrane association as a critical element in its clamping function and in vivo interactions with SNARE proteins.

As shown in Figure 8, our working model suggests that membrane association of CPX promotes its interactions with SNARE proteins and is required for the CPX clamping function. At wild-type synapses (Fig. 8A), CPX is associated with presynaptic membranes, including clear localization to SVs as well as the $\mathrm{AZ}$ where neurotransmitter release sites reside (Fig. 5). In contrast, the $c p X^{1257}$ mutant (Fig. 8B) exhibits an altered CPX distribution reflecting loss of membrane-associated $\mathrm{CPX}$ in general but retention of CPX localization at the AZ (Fig. 5). This altered distribution is also associated with loss of CPX membrane targeting in partitioning assays (Fig. 6), a drastic reduction in CPX-SNARE binding interactions (Fig. 7) and loss of the CPX clamping function (Fig. 3 and 4) in the $c p X^{1257}$ mutant. Notably, the activation role for CPX in evoked neurotransmitter release is retained in this mutant (Fig. 3 and 4), which likely reflects retention of CPX localization to AZs and its interactions with a subset of SNARE proteins localized at neurotransmitter release sites. Thus our working model suggests that AZ-associated and non-AZ membrane-associated 
pools of CPX are localized by different mechanisms, participate in different molecular interactions and make distinct functional contributions to spontaneous and evoked release.

The preceding model extends and modifies the conclusions of previous work and complements very recent studies of CPX in C. elegans (Wragg et al., 2013). Although the present findings first demonstrate that a farnesylated form of CPX is distributed into two separable CPX pools within the presynaptic terminal, previous work has demonstrated that CPX farnesylation is required for localization to synaptic regions of the axon and association with membranes in biochemical fractionation experiments (Reim et al., 2005). Notably, it was recently shown that a non-farnesylated form of CPX in C. elegans associates with SVs through its $\mathrm{C}$-terminus and that $\mathrm{SV}$ association is required for the CPX clamping function (Wragg et al., 2013). Other related studies have mapped the CPX clamping function to its C terminus (Kaeser-Woo et al., 2012; Martin et al., 2011) and the CaaX motif (Buhl et al., 2013; Cho et al., 2010; Xue et al., 2009). A recent study in Drosophila recovered a $c p x$ mutant, $c p x^{572}$, lacking the last 25 amino acids of the C-terminus and reported an increase in mini frequency and a modest reduction in evoked neurotransmitter release at larval neuromuscular synapses. This phenotype resembles that described here for the $c p x^{1257}$ mutant, which lacks only the last C-terminal amino acid, however $c p x^{1257}$ exhibited wildtype evoked release (Fig. 4). The state of CPX lipid modification was not addressed in the previous study nor was investigation of changes in CPX-SNARE interactions resulting from the mutation. A change in CPX distribution was reported for CPX ${ }^{572}$ at larval neuromuscular synapses, however the relationship of $\mathrm{CPX}$ to the $\mathrm{AZ}$ was not clear in this study (Buhl et al., 2013). For comparison, immunocytochemistry of $c p x^{1257}$ mutant larvae was carried out to examine the presynaptic localization of CPX (Fig. S2). The CPX ${ }^{1257}$ distribution resembled that described previously for $\mathrm{CPX}^{572}$, characterized by a diffuse CPX signal which was only partially colocalized with AZs. The differences observed between adult and larval neuromuscular synapses may reflect their distinctive morphologies. In contrast to the high density of AZs within presynaptic boutons of larval neuromuscular synapses, adult neuromuscular synapses are characterized by small presynaptic boutons which typically contain one or two active zones. The latter morphology can be advantageous for resolving protein localization to the AZ and periactive zone regions (Kawasaki et al., 2011; Kawasaki and Ordway, 2009). The following discussion of our findings will focus on the relationship of CPX membrane association to its binding interactions with SNAREs, as well as the molecular mechanisms of the CPX clamping function.

\section{The relationship of CPX membrane targeting to its interactions with SNARE proteins}

The present study demonstrates for the first time that CPX is localized to the AZ and complements our previous work demonstrating AZ localization of the t-SNARE proteins, SYX and SNAP-25 (Kawasaki and Ordway, 2009). These findings are consistent with models suggesting that CPX interacts with t-SNAREs as well as with trans-SNARE complexes occurring between primed synaptic vesicles and the $\mathrm{AZ}$ region of the presynaptic plasma membrane (Chen et al., 2002; Jahn and Scheller, 2006; McMahon et al., 1995; Weninger et al., 2008). Furthermore, CPX is also localized more generally to presynaptic membranes, including synaptic vesicles, presumably in the absence of trans-SNARE complexes. Surprisingly, loss of the non-AZ membrane localization in the $c p x^{1257}$ mutant (Figs. 5 and 6) produced a drastic reduction in CPX interactions with both v- and t-SNAREs (Fig. 7) despite the persistence of CPX localization at the AZ (Fig. 5). Thus it appears $\mathrm{CPX}^{1257}$ retains its ability to function in evoked neurotransmitter release by binding SNAREs at the AZ through a farnesylation-independent mechanism, while losing its ability to bind SNAREs more generally through its membrane association. These results indicate that the preponderance of CPX-SNARE interactions are not restricted to the AZ but rather occur more generally with plasma membrane t-SNAREs and synaptic vesicle v-SNAREs. 
Although the current study does not rule out the possibility that CPX ${ }^{1257}$ exhibits a change in binding affinity for SNAREs located outside of the AZ, we favor the interpretation that broader CPX-SNARE interactions are promoted by membrane-association of farnesylated CPX. It is of interest to note the farnesylation dependence of both CPX localization to SVs and CPX interaction with the v-SNARE, NSYB. However, because NSYB is also present on the plasma membrane (Fernández-Alfonso et al., 2006; Kawasaki and Ordway, 2009; Taubenblatt et al., 1999), it remains unclear whether the farnesylation-dependent interactions of CPX with NSYB occur strictly on SVs.

\section{The molecular mechanisms of the CPX clamping function}

The relationship of the preceding molecular interactions to CPX function may be observed in parallel electrophysiological analysis of the $c p x^{1257}$ mutant (Fig. 3 and 4). The finding that evoked neurotransmitter release is normal in $c p x^{1257}$ suggests that normal AZ localization of CPX in this mutant preserves a minor component of the total CPX-SNARE interactions (see Fig. 7), which occurs at the $\mathrm{AZ}$ and is sufficient to support evoked release. In contrast, the loss of CPX clamping in this mutant suggests that broader membrane localization of CPX, which is associated with the preponderance of CPX-SNARE interactions occurring outside of the AZ, is critical to clamping. These observations raise the possibility that farnesylation-dependent pre-association of CPX with SNARE proteins, which occurs predominantly outside of the AZ on either the synaptic vesicle or plasma membrane, may contribute to the clamping mechanism. How such a role for CPX membrane association might relate to the mechanism of accessory helix function in clamping remains an interesting question.

It is of interest to consider the relevance of these mechanisms to CPX isoforms which are not farnesylated, such as mammalian CPX1 and 2 as well as $C$. elegans CPX-1 (Brose, 2008). Previous studies indicated that non-farnesylated isoforms of mammalian CPX also exhibit phospholipid binding activity through a C-terminal amphipathic helix (Seiler et al., 2009) which is necessary for the CPX clamping function (Kaeser-Woo et al., 2012). Recent work in C. elegans (Wragg et al., 2013) builds on these previous findings to show that SVlocalization of a non-farnesylated CPX isoform is required for the clamping function in a manner analogous to that reported here for farnesylated CPX. It will be of interest to examine the role of distinct localization mechanisms in determining similar subcellular distributions and functions for farnesylated and non-farnesylated CPX isoforms. In Drosophila, a non-farnesylated isoform of CPX was shown to be functional in CPX activation and clamping (Huntwork and Littleton, 2007). While the present work focused on farnesylated isoforms of Drosophila CPX, which are predominant in the nervous system (Fig. 2), this non-farnesylated isoform (DmCPX-E; Fig. 2) exhibited a similar subcellular distribution when expressed within the presynaptic terminal (not shown). These observations suggest that the clamping function of CPX may depend upon its membrane targeting in general, rather than interactions specific to farnesylated forms.

\section{Supplementary Material}

Refer to Web version on PubMed Central for supplementary material.

\section{Acknowledgments}

A $c p x$ null mutant stock, a $U A S$-cpx transgenic line and an anti-CPX antibody were generously provided by Troy Littleton (MIT, Cambridge, MA). We are also grateful to Noreen Reist (Colorado State University, Fort Collins, CO) and David Deitcher (Cornell University, Ithaca, NY) for providing anti-SYT and anti-SNAP25 antibodies, respectively. We thank Richard Ordway (Penn State University) for his continuous encouragement and invaluable discussion throughout this work. This study was supported by National Institutes of Health Grant R21MH085199-02. 


\section{Abbreviations}

$\begin{array}{ll}\text { AZ } & \text { active zone } \\ \text { BRP } & \text { BRUCHPILOT } \\ \text { CPX } & \text { COMPLEXIN } \\ \text { DLM } & \text { dorsal longitudinal flight muscle } \\ \text { EPSC } & \text { excitatory postsynaptic current } \\ \text { mEPSC } & \text { miniature excitatory postsynaptic current } \\ \text { mEPSP } & \text { miniature excitatory postsynaptic potential } \\ \text { NSYB } & \text { neuronal-SYNAPTOBREVIN } \\ \text { SV } & \text { synaptic vesicle } \\ \text { SYT } & \text { SYNAPTOTAGMIN } \\ \text { SYX } & \text { SYNTAXIN }\end{array}$

\section{REFERENCES}

Bordier C. Phase separation of integral membrane proteins in Triton X-114 solution. J. Biol. Chem. 1981; 256:1604-1607. [PubMed: 6257680]

Bowen ME, Weninger K, Ernst J, Chu S, Brunger AT. Single-molecule studies of synaptotagmin and complexin binding to the SNARE complex. Biophys. J. 2005; 89:690-702. [PubMed: 15821166]

Bracher A, Kadlec J, Betz H, Weissenhorn W. X-ray structure of a neuronal complexin-SNARE complex from squid. J. Biol. Chem. 2002; 277:26517-22623. [PubMed: 12004067]

Brand AH, Perrimon N. Targeted gene expression as a means of altering cell fates and generating dominant phenotypes. Development. 1993; 118:401-415. [PubMed: 8223268]

Brose N. For better or for worse: complexins regulate SNARE function and vesicle fusion. Traffic. 2008; 9:1403-1413. [PubMed: 18445121]

Buhl LK, Jorquera RA, Akbergenova Y, Huntwork-Rodriguez S, Volfson D, Littleton JT. Differential regulation of evoked and spontaneous neurotransmitter release by $\mathrm{C}$-terminal modifications of complexin. Mol. Cell. Neurosci. 2013; 52:161-172. [PubMed: 23159779]

Chen X, Tomchick DR, Kovrigin E, Araç D, Machius M, Südhof TC, Rizo J. Three-dimensional structure of the complexin/SNARE complex. Neuron. 2002; 33:397-409. [PubMed: 11832227]

Cho RW, Song Y, Littleton JT. Comparative analysis of Drosophila and mammalian complexins as fusion clamps and facilitators of neurotransmitter release. Mol. Cell. Neurosci. 2010; 45:389-397. [PubMed: 20678575]

Dellinger BB, Felling R, Ordway RW. Genetic modifiers of the Drosophila NSF mutant, comatose, include a temperature-sensitive paralytic allele of the calcium channel a1 subunit gene, cacophony. Genetics. 2000; 155:203-211. [PubMed: 10790395]

Fernández-Alfonso T, Kwan R, Ryan TA. Synaptic vesicles interchange their membrane proteins with a large surface reservoir during recycling. Neuron. 2006; 51:179-186. [PubMed: 16846853]

Hobson RJ, Liu Q, Watanabe S, Jorgensen EM. Complexin maintains vesicles in the primed state in C. elegans. Curr. Biol. 2011; 21:106-113. [PubMed: 21215631]

Huntwork S, Littleton JT. A complexin fusion clamp regulates spontaneous neurotransmitter release and synaptic growth. Nat. Neurosci. 2007; 10:1235-1237. [PubMed: 17873870]

Ishizuka T, Saisu H, Odani S, Abe T. Synaphin: a protein associated with the docking/fusion complex in presynaptic terminals. Biochem. Biophys. Res. Commun. 1995; 213:1107-1114. [PubMed: 7654227]

Jahn R, Scheller RH. SNAREs-engines for membrane fusion. Nat. Rev. Mol. Cell Biol. 2006; 7:631643. [PubMed: 16912714] 
Jorquera RA, Huntwork-Rodriguez S, Akbergenova Y, Cho RW, Littleton JT. Complexin controls spontaneous and evoked neurotransmitter release by regulating the timing and properties of synaptotagmin activity. J. Neurosci. 2012; 32:18234-18245. [PubMed: 23238737]

Kaeser-Woo YJ, Yang X, Sudhof TC. C-terminal complexin sequence is selectively required for clamping and priming but not for Ca2+ triggering of synaptic exocytosis. J. Neurosci. 2012; 32:2877-2885. [PubMed: 22357870]

Kawasaki F, Iyer J, Posey LL, Sun CE, Mammen SE, Yan H, Ordway RW. The DISABLED protein functions in CLATHRIN-mediated synaptic vesicle endocytosis and exoendocytic coupling at the active zone. Proc. Natl. Acad. Sci. USA. 2011; 108:E222-229. [PubMed: 21606364]

Kawasaki F, Ordway RW. Molecular mechanisms determining conserved properties of short-term synaptic depression revealed in NSF and SNAP-25 conditional mutants. Proc. Natl. Acad. Sci. USA. 2009; 106:14658-14663. [PubMed: 19706552]

Kawasaki F, Zou B, Xu X, Ordway RW. Active zone localization of presynaptic calcium channels encoded by the cacophony. J. Neurosci. 2004; 24:282-285. [PubMed: 14715960]

Li Y, Augustine GJ, Weninger K. Kinetics of complexin binding to the SNARE complex: correcting single molecule FRET measurements for hidden events. Biophys. J. 2007; 93:2178-2187. [PubMed: 17513363]

Lutas A, Wahlmark CJ, Acharjee S, Kawasaki F. Genetic analysis in Drosophila reveals a role for the mitochondrial protein, P32, in synaptic transmission. G3. 2012; 2:59-69. [PubMed: 22384382]

Martin JA, Hu Z, Fenz KM, Fernandez J, Dittman JS. Complexin has opposite effects on two modes of synaptic vesicle fusion. Curr. Biol. 2011; 21:97-105. [PubMed: 21215634]

Mathias RA, Chen YS, Kapp EA, Greening DW, Mathivanan S, Simpson RJ. Triton X-114 phase separation in the isolation and purification of mouse liver microsomal membrane proteins. Methods. 2011; 54:396-406. [PubMed: 21272644]

Maximov A, Tang J, Yang X, Pang ZP, Südhof TC. Complexin controls the force transfer from SNARE complexes to membranes in fusion. Science. 2009; 323:516-521. [PubMed: 19164751]

McMahon HT, Missler M, Li C, Südhof TC. Complexins: cytosolic proteins that regulate SNAP receptor function. Cell. 1995; 83:111-119. [PubMed: 7553862]

Neher E. Complexin: does it deserve its name? Neuron. 2010; 68:803-806. [PubMed: 21144993]

Omer CA, Gibbs JB. Protein prenylation in eukaryotic microorganisms: genetics, biology and biochemistry. Molecular microbiology. 1994; 11:219-225. [PubMed: 8170384]

Pabst S, Margittai M, Vainius D, Langen R, Jahn R, Fasshauer D. Rapid and selective binding to the synaptic SNARE complex suggests a modulatory role of complexins in neuroexocytosis. J. Biol. Chem. 2002; 277:7838-7848. [PubMed: 11751907]

Price CT, Al-Quadan T, Santic M, Jones SC, Abu Kwaik Y. Exploitation of conserved eukaryotic host cell farnesylation machinery by an F-box effector of Legionella pneumophila. J. Exp. Med. 2010; 207:1713-1726. [PubMed: 20660614]

Reim K, Mansour M, Varoqueaux F, McMahon HT, Südhof TC, Brose N, Rosenmund C. Complexins regulate a late step in $\mathrm{Ca}^{2+}$-dependent neurotransmitter release. Neuron. 2001; 104:71-81.

Reim K, Wegmeyer H, Brandstätter JH, Xue M, Rosenmund C, Dresbach T, Hofmann K, Brose N. Structurally and functionally unique complexins at retinal ribbon synapses. J. Cell Biol. 2005; 169:669-680. [PubMed: 15911881]

Reinicke AT, Hutchinson JL, Magee AI, Mastroeni P, Trowsdale J, Kelly AP. A Salmonella typhimurium effector protein SifA is modified by host cell prenylation and S-acylation machinery. J. Biol. Chem. 2005; 280:14620-14627. [PubMed: 15710609]

Resh MD. Trafficking and signaling by fatty-acylated and prenylated proteins. Nat. Chem. Biol. 2006; 2:584-590. [PubMed: 17051234]

Rizo J, Rosenmund C. Synaptic vesicle fusion. Nat. Struct. Mol. Biol. 2008; 15:665-674. [PubMed: 18618940]

Seiler F, Malsam J, Krause JM, Sollner TH. A role of complexin-lipid interactions in membrane fusion. FEBS Lett. 2009; 583:2343-2348. [PubMed: 19540234]

Stein A, Jahn R. Complexins living up to their name--new light on their role in exocytosis. Neuron. 2009; 64:295-297. [PubMed: 19914176] 
Strenzke N, Chanda S, Kopp-Scheinpflug C, Khimich D, Reim K, Bulankina AV, Neef A, Wolf F, Brose N, Xu-Friedman MA, Moser T. Complexin-I is required for high-fidelity transmission at the endbulb of Held auditory synapse. J. Neurosci. 2009; 29:7991-8004. [PubMed: 19553439]

Südhof TC, Rothman JE. Membrane fusion: grappling with SNARE and SM proteins. Science. 2009; 323:474-477. [PubMed: 19164740]

Tang J, Maximov A, Shin OH, Dai H, Rizo J, Südhof TC. A complexin/synaptotagmin 1 switch controls fast synaptic vesicle exocytosis. Cell. 2006; 126:1175-1187. [PubMed: 16990140]

Taubenblatt P, Dedieu JC, Gulik-Krzywicki T, Morel N. VAMP (synaptobrevin) is present in the plasma membrane of nerve terminals. J. Cell Sci. 1999; 112:3559-3567. [PubMed: 10504304]

Tolar LA, Pallanck L. NSF function in neurotransmitter release involves rearrangement of the SNARE complex downstream of synaptic vesicle docking. J. Neurosci. 1998; 18:10250-10256. [PubMed: 9852562]

Wang L, Coppel RL. Triton X-114 phase partitioning for antigen characterization. Methods Mol. Med. 2002; 72:581-585. [PubMed: 12125157]

Weninger K, Bowen ME, Choi UB, Chu S, Brunger AT. Accessory proteins stabilize the acceptor complex for synaptobrevin, the 1:1 syntaxin/SNAP-25 complex. Structure. 2008; 16:308-320. [PubMed: 18275821]

Wragg RT, Snead D, Dong Y, Ramlall TF, Menon I, Bai J, Eliezer D, Dittman JS. Synaptic vesicles position complexin to block spontaneous fusion. Neuron. 2013; 77:323-334. [PubMed: 23352168]

Wu Y, Kawasaki F, Ordway RW. Properties of short-term synaptic depression at larval neuromuscular synapses in wild-type and temperature-sensitive paralytic mutants of Drosophila. J. Neurophysiol. 2005; 93:2396-2405. [PubMed: 15845998]

Xue M, Craig TK, Xu J, Chao HT, Rizo J, Rosenmund C. Binding of the complexin N terminus to the SNARE complex potentiates synaptic-vesicle fusogenicity. Nat. Struct. Mol. Biol. 2010; 17:568575. [PubMed: 20400951]

Xue M, Lin YQ, Pan H, Reim K, Deng H, Bellen HJ, Rosenmund C. Tilting the balance between facilitatory and inhibitory functions of mammalian and Drosophila Complexins orchestrates synaptic vesicle exocytosis. Neuron. 2009; 64:367-380. [PubMed: 19914185]

Xue M, Reim K, Chen X, Chao HT, Deng H, Rizo J, Brose N, Rosenmund C. Distinct domains of complexin I differentially regulate neurotransmitter release. Nat. Struct. Mol. Biol. 2007; 14:949_ 958. [PubMed: 17828276]

Yang X, Kaeser-Woo YJ, Pang ZP, Xu W, Südhof TC. Complexin clamps asynchronous release by blocking a secondary $\mathrm{Ca}^{2+}$ sensor via its accessory a helix. Neuron. 2010; 68:907-920. [PubMed: 21145004]

Yu W, Kawasaki F, Ordway RW. Activity-dependent interactions of NSF and SNAP at living synapses. Mol. Cell. Neurosci. 2011; 47:19-27. [PubMed: 21316453]

Zou B, Yan H, Kawasaki F, Ordway RW. MAP1 structural organization in Drosophila: In vivo analysis of FUTSCH reveals heavy and light chain subunits generated by proteolytic processing at a conserved cleavage site. Biochem. J. 2008; 414:63-71. [PubMed: 18419581] 


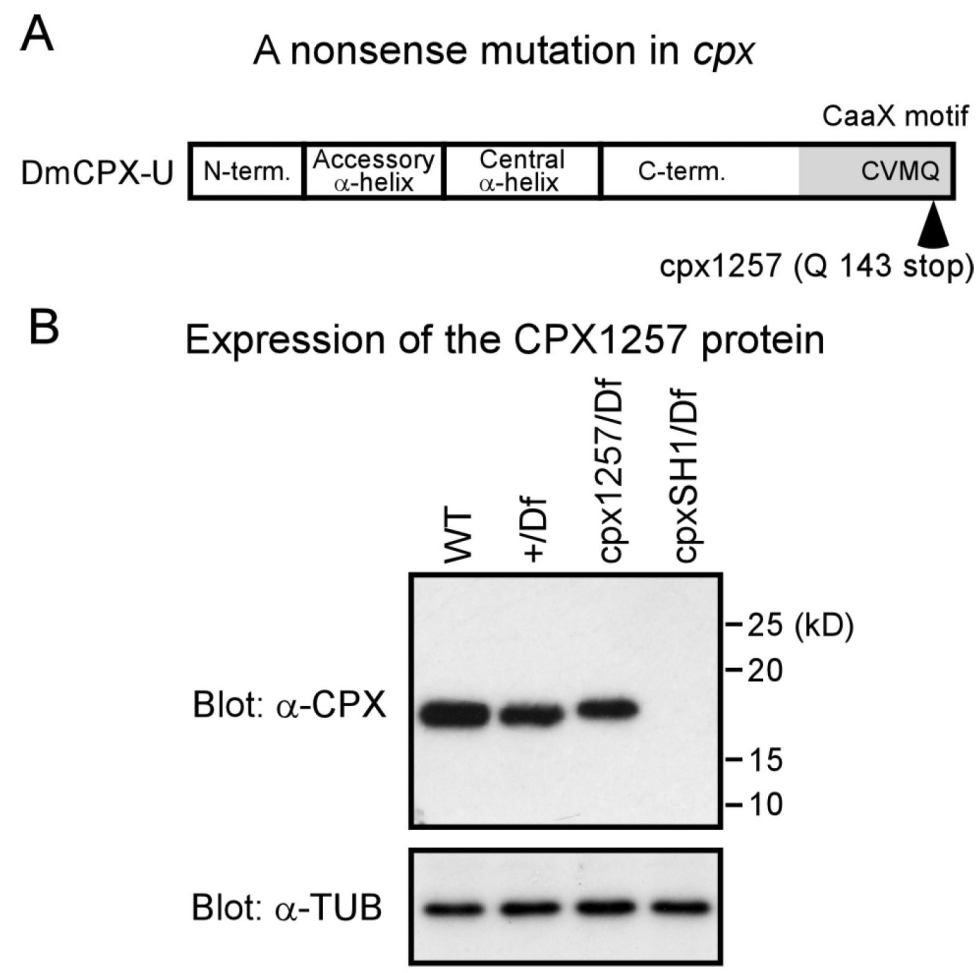

Figure 1. A New cpx mutant, $c p x^{1257}$

(A) Schematic of Drosophila CPX (isoform DmCPX-U) and the nonsense mutation in $c p x^{1257}$. The $c p x^{1257}$ mutation removes the last $\mathrm{C}$-terminal amino acid of CPX, corresponding to the $\mathrm{X}$ in the $\mathrm{CaaX}$ motif required for farnesylation. This mutation is isoform-specific in that it occurs in an alternative exon encoding the C-terminus of CPX (highlighted in gray; see also Fig. 2). The domain organization shown was adapted from a previous study (Xue et al., 2007). The accession number for DmCPX-U is AY121629. (B) Western analysis of $c x^{1257}$. Western blot of fly head homogenates prepared from wild type (WT) and $c p x$ hemizygotes heterozygous for $D f(3 R)$ Exel6140(Df) which removes the $c p x$ locus, as well as $c p x^{1257} / D f$ and $c p x^{S H 1} / D f$. Although $C P X^{1257}$ migrates at a slightly higher relative molecular mass in comparison to wild-type CPX, the levels in the $c p x^{1257 / D f \text { and }+/}$ $D f$ samples are similar, indicating normal CPX expression in $c p x^{1257}$. CPX is absent in $c p x^{S H 1} / D f$ hemizygotes. Tubulin (TUB) was used as an internal loading control. 
A

Drosophila CPX isoforms generated by alternative splicing from one cpx gene

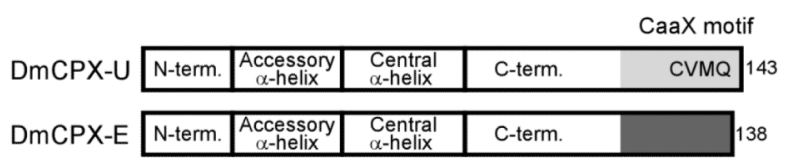

B

Predominant brain isoforms of Drosophila CPX contain the CAAX farnesylation motif

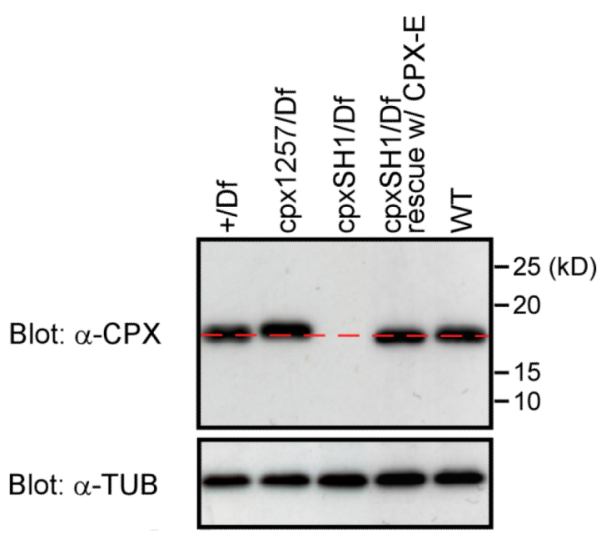

Figure 2. CPX isoforms contain distinct C-terminal domains

(A) Two Drosophila CPX (DmCPX) isoforms generated by alternative splicing from the single $c p x$ gene. A main difference between them is that the $\mathrm{C}$-terminus (shown in different shades of gray) is encoded by two different alternative exons. DmCPX isoforms $U$ and E, respectively, represent isoforms predicted to be farnesylated (CaaX containing) or not farnesylated (CaaX lacking). The exon containing the CaaX motif is present in most isoforms (http://www.flybase.org, see also MATERIALS AND METHODS SectionRelevant information about cpx alternative exons and splice variants). (B) Predominant brain isoforms of Drosophila CPX contain the CaaX farnesylation motif. Western analysis of fly head homogenates prepared from WT, $c p_{x}$ hemizygotes heterozygotes for $D f(3 R) E x e 16140$ (+/Df), $c p x^{1257} / D f, c p x^{S H 1} / D f$ and $c p x^{S H 1} / D f$ rescued by neural expression of the wild-type CPX isoform E. Rescue of $c p x^{S H 1}$ was carried out in Appl-GAL4;;UAS$c p x c p x$ SH1 $/ D f(3 R) E x e 16140$ flies. The predominance of CaaX-containing CPX isoforms in the Drosophila brain is inferred from the following observations. First, CPX-E migrates at a slightly lower relative molecular mass in comparison to wild-type CPX from $+/ D f$ and WT flies and does not appear to be detected in head homogenates containing endogenous CPX. Second, the altered migration of $\mathrm{CPX}^{1257}$ appears to shift all of the detectable endogenous CPX signal. Tubulin (TUB) was used as an internal loading control. 


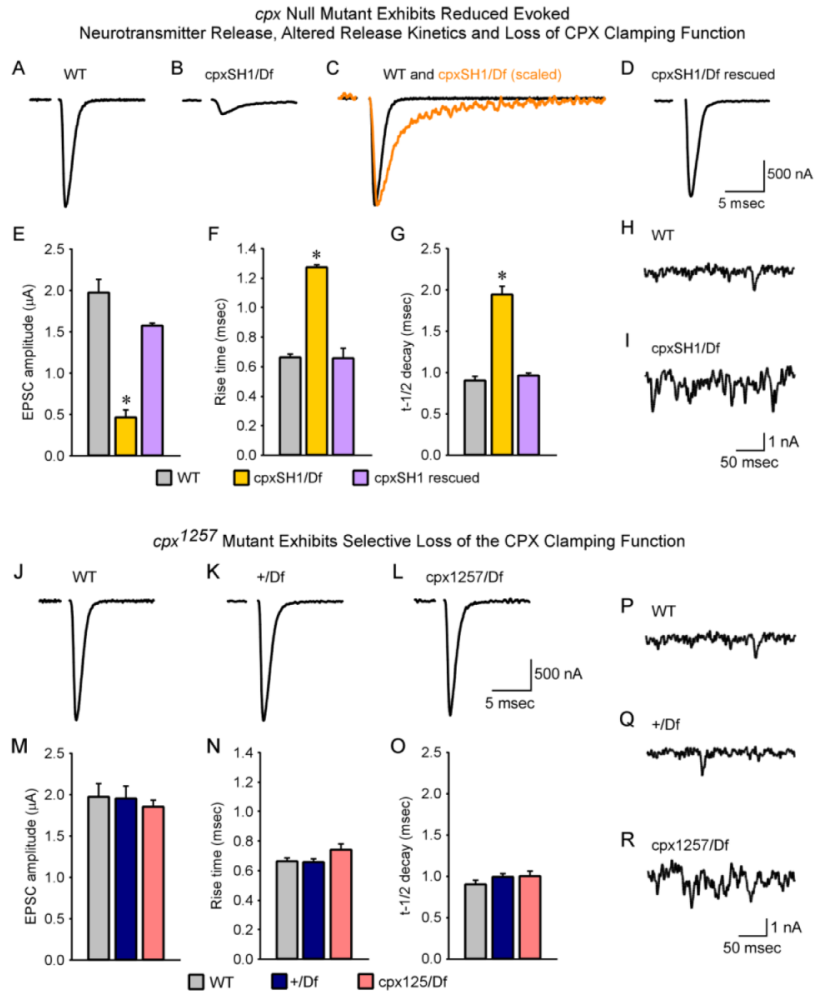

Figure 3. Electrophysiological analysis of $c p x$ mutant phenotypes at DLM neuromuscular synapses

(A-I) The $c p x$ null mutant, $c p x^{S H 1}$, exhibits reduced evoked neurotransmitter release, altered release kinetics and loss of the CPX clamping function. (A, B) Representative excitatory postsynaptic current (EPSC) recordings from dorsal longitudinal flight muscle (DLM) neuromuscular synapses of wild type (WT) (A) and $c p x S H 1 / D f(3 R)$ Exel6140 (cpxSH1/Df) (B). cpx null mutant (cpxSH1/Df) synapses exhibit a marked reduction in the EPSC amplitude. (C) The $c p x^{S H 1}$ null mutant exhibits a slowing of the rise and decay times of the EPSC waveform. The EPSC in WT (black) was superimposed with a scaled version of the EPSC in cpxSH1/Df (gray). (D) The synaptic phenotypes in $c p x^{S H 1}$ were rescued by neural (presynaptic) expression of the wild-type CPX protein. Rescue of $c p x^{S H 1}$ was carried out in Appl-GAL4;UAS-EGFP-cpx/+;cpx SH1/Df(3R)Exel6140 flies. With respect to WT, the $c p x^{S H 1}$ mutant exhibited an decrease in EPSC amplitude (E) as well as an increase in rise (time-to-peak) (F) and in decay (t1/2) (G) kinetics. The EPSC amplitude in WT, cpxSH1/Df and cpxSH1 rescued was $1.97 \pm 0.16 \mu \mathrm{A}(\mathrm{n}=4), 0.46 \pm 0.08 \mu \mathrm{A}(\mathrm{n}=4)$ and 1.57 $\pm 0.03 \mu \mathrm{A}(\mathrm{n}=3)$, respectively. The EPSC rise time in WT, cpxSH1/Df and cpxSH1 rescued was $0.66 \pm 0.02 \mathrm{msec}(n=4), 1.27 \pm 0.01 \mathrm{msec}(n=4)$ and $0.65 \pm 0.07 \mathrm{msec}(n=3)$, respectively. The EPSC decay time in WT, cpxSH1/Df and cpxSH1 rescued was $0.90 \pm 0.05$ msec $(n=4), 1.94 \pm 0.10 \mathrm{msec}(n=4)$ and $0.96 \pm 0.02 \mathrm{msec}(\mathrm{n}=3)$, respectively. $(\mathrm{H}, \mathrm{I})$

Representative miniature EPSC (mEPSC) recordings from DLM neuromuscular synapses of WT (H) and cpxSH1/Df (I). The mEPSC frequency in $c p x S H 1 / D f$ was severely elevated. (JR) $c p x^{1257}$ exhibits selective loss of the CPX clamping function. (J, K and L) Representative EPSC recordings from DLM neuromuscular synapses of WT $(\mathrm{J}), c p x$ hemizygotes $[+/$ $D f(3 R) E x e l 6140$ (+/Df)] (K) and $c p x^{1257} / D f(3 R) E x e l 6140$ (cpx1257/Df) (L). (M, N and O) No change in the EPSC amplitude or waveform was observed in the $c p x^{1257}$ mutant. The data for WT is the same as shown in E, F and G. The EPSC amplitude in +/Df and cpx1257/ Df was $1.95 \pm 0.16 \mu \mathrm{A}(\mathrm{n}=4)$ and $1.85 \pm 0.08 \mu \mathrm{A}(\mathrm{n}=5)$, respectively. The EPSC rise time in +/Df and cpx1257/Df was $0.65 \pm 0.02 \mathrm{msec}(\mathrm{n}=4)$ and $0.74 \pm 0.04 \mathrm{msec}(\mathrm{n}=5)$, respectively. 
The EPSC decay time in +/Df and cpx1257/Df was $0.99 \pm 0.03 \mathrm{msec}(\mathrm{n}=4)$ and $1.00 \pm 0.05$ msec ( $\mathrm{n}=5$ ), respectively. (P, Q and $\mathrm{R})$ Representative miniature EPSC (mEPSC) recordings from DLM neuromuscular synapses of WT (P), +/Df (Q) and cpx ${ }^{1257} / \mathrm{Df}(\mathrm{R})$. The mEPSC frequency in $c p x^{1257} / D f$ was severely elevated. Error bars indicate SEM and asterisks denote statistical significance at $p \unlhd 0.05$. 

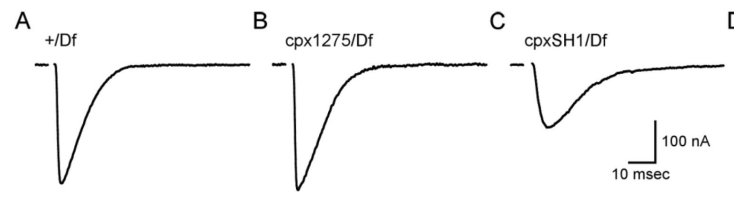

D $+/ \mathrm{Df}$ and cpxSH1/Df (scaled)

E

$\mathrm{F}$

G
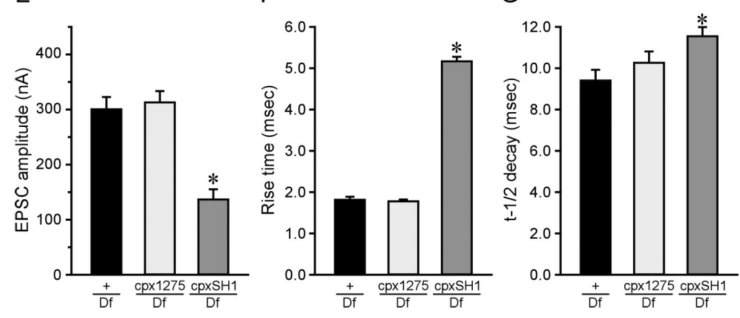

$\mathrm{H}$
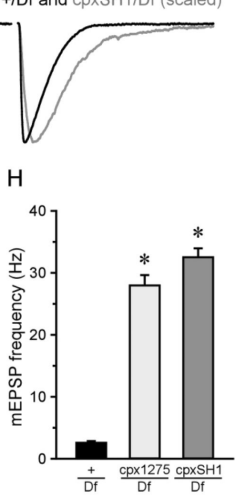

Figure 4. Electrophysiogical analysis of $\operatorname{cpx}$ mutant phenotypes at larval neuromuscular synapses

The $c p x$ null mutant, $c p x$ SH1, exhibits reduced evoked neurotransmitter release, altered release kinetics and loss of the CPX clamping function, whereas $c p x^{1257}$ exhibits selective loss of the CPX clamping function. (A-D) Representative EPSC recordings from DLM neuromuscular synapses of $c p x$ hemizygotes [+/Df(3R)Exel6140(+/Df)] (A), $c p x^{1257}$ / $D f(3 R) E x e 16140$ (cpx1257/Df) (B) and $c p x{ }^{S H 1} / D f(3 R) E x e l 6140$ (cpxSH1/Df). (D) The $c p x^{S H 1}$ null mutant exhibits a slowing of the rise and decay times of the EPSC waveform. The EPSC in $c p x$ hemizygotes (+/Df, black) was superimposed with a scaled version of the EPSC in $\mathrm{cpx}^{\mathrm{SH} 1} / \mathrm{Df}$ (gray).(E, F and G) No change in the EPSC amplitude or waveform was observed in the $c p x^{1257}$ mutant (cpx1257/Df). In contrast, the $c p x$ null mutant (cpxSH1/Df) exhibited a marked reduction in the EPSC amplitude and a slowing of the rise and decay times of the EPSC waveform. The EPSC amplitude in +/Df, cpx1257/Df and cpxSH1/Df was $299.8 \pm 44.73 \mathrm{nA}(\mathrm{n}=4), 312.3 \pm 44.10 \mathrm{nA}(\mathrm{n}=4)$ and $135.6 \pm 41.93 \mathrm{nA}(\mathrm{n}=5)$, respectively. The EPSC rise time in +/Df, cpx1257/Df and cpxSH1/Df was $1.82 \pm 0.14 \mathrm{msec}$ $(\mathrm{n}=4), 1.78 \pm 0.08 \mathrm{msec}(\mathrm{n}=4)$ and $5.17 \pm 0.25 \mathrm{msec}(\mathrm{n}=5)$, respectively. The EPSC decay time in +/Df, cpx1257/Df and cpxSH1/Df was 9.37 $\pm 1.05 \mathrm{msec}(\mathrm{n}=4), 10.24 \pm 1.12 \mathrm{msec}$ $(\mathrm{n}=4)$ and $11.53 \pm 0.96 \mathrm{msec}(\mathrm{n}=5)$, respectively. $(\mathrm{H})$ The mEPSP frequency in $c p x^{1275} / D f$ and $c p_{x} S H 1 / D f$ was severely elevated. The mEPSP frequency in +/Df, cpx1257/Df and cpxSH1/Df was $2.8 \pm 0.18 \mathrm{~Hz}(\mathrm{n}=4), 28.1 \pm 1.56 \mathrm{~Hz}(\mathrm{n}=4)$ and $32.7 \pm 1.33 \mathrm{~Hz}(\mathrm{n}=4)$, respectively. 

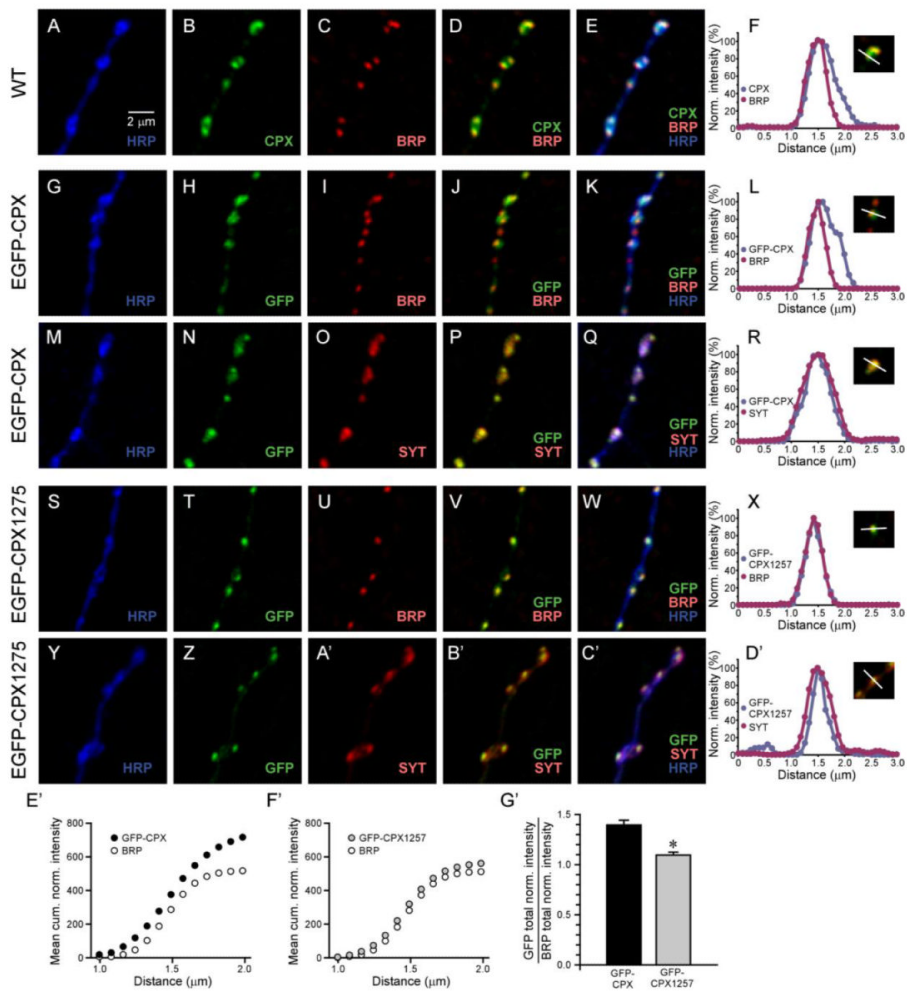

Figure 5. Presynaptic localization of wild-type CPX and mutant CPX ${ }^{1257}$ at DLM neuromuscular synapses

(A-R) CPX is highly localized at the AZ and also exhibits a broader pattern which is colocalized with synaptic vesicles. Confocal immunofluorescence and native EGFP fluorescence images of DLM neuromuscular synapses showing expression of endogenous CPX (A-E), neuronal (presynaptic) expression of EGFP-CPX (G-K and M-Q) and either the active zone (AZ) marker, BRUCHPILOT (BRP) (A-E and G-K) or the synaptic vesicle marker, SYNAPTOTAGMIN (SYT) (M-Q). (F, L) Comparisons of pixel intensity profiles for CPX (F) or EGFP-CPX (L) with those for BRP. (R) Comparison of pixel intensity profile for EGFP-CPX with that for SYT. Insets in panels F, L and R correspond to boutons shown in panels $\mathrm{D}, \mathrm{J}$ and $\mathrm{P}$, respectively. A white line shown in each inset image designates a line of pixels whose intensities were normalized to the maximum pixel intensity and plotted as a function of distance along the line. The distribution of endogenous CPX and EGFP-CPX relative to BRP indicated strong CPX localization to AZs and also a more diffuse pattern which is colocalized with the synaptic vesicle marker, SYT. Anti-HRP labels the neuronal plasma membrane. Native EGFP fluorescence is denoted as GFP. (S-D') CPX localization to the AZ, but not broader membrane localization to SVs, is retained in the $c p x^{1257}$ mutant. Confocal immunofluorescence and native EGFP fluorescence images of DLM neuromuscular synapses showing neuronal expression of EGFP-CPX ${ }^{1257}$. EGFP$\mathrm{CPX}^{1257}$ was highly localized at AZs (S-X) as observed for wild-type EGFP-CPX, however it lacked the broader distribution which colocalized with the synaptic vesicle marker, SYT (Y-D'). Additional experiments to eliminate any possible competition between the EGFP$\mathrm{CPX}^{1257}$ fusion protein and endogenous wild-type CPX were carried out in acpx null mutant background and produced similar results (Fig. S1). (X and D') Comparisons of pixel intensity profiles for EGFP-CPX ${ }^{1257}$ with those for BRP (X) or SYT (D'). Insets in panels X and $D^{\prime}$ correspond to boutons shown in panels $V$ and $B^{\prime}$, respectively. (E', F') Cumulative intensity plots. For each condition, normalized intensity profiles such as those shown in panels $\mathrm{L}$ and $\mathrm{X}$ were averaged to generate mean normalized pixel intensities for each pixel 
along the line. A one micron segment of the line, centered on the maximum BRP intensity, was selected for further analysis. Cumulative plots of mean normalized pixel intensities as a function of distance along the line segment were generated to compare the distribution of the AZ marker, BRP, to that of GFP-CPX (E') or GFP-CPX ${ }^{1257}$ (F').

For each genotype, GFP-CPX and GFP-CPX ${ }^{1257}$, this analysis included four preparations and a total of 16 or 22 images, respectively. ( $\left.G^{\prime}\right)$ Comparison of total normalized intensities (maximal cumulative values in panels $\mathrm{E}^{\prime}$ and $\mathrm{F}^{\prime}$ ) expressed as a ratio of the total normalized intensity for either EGFP-CPX or EGFP-CPX ${ }^{1257}$ to that for BRP. For EGFP-CPX, a ratio of $1.39 \pm 0.04(n=16)$ was observed, reflecting the broader distribution of EGFP-CPX in comparison to BRP. In contrast, the corresponding value for EGFP-CPX ${ }^{1257}$ was $1.10 \pm$ $0.02(n=22)$ indicating the distribution of EGFP-CPX ${ }^{1257}$ is more similar to that of BRP. These values were significantly different $(*)$ with a $p$ value of $6.6 \times 10^{-8}$. 


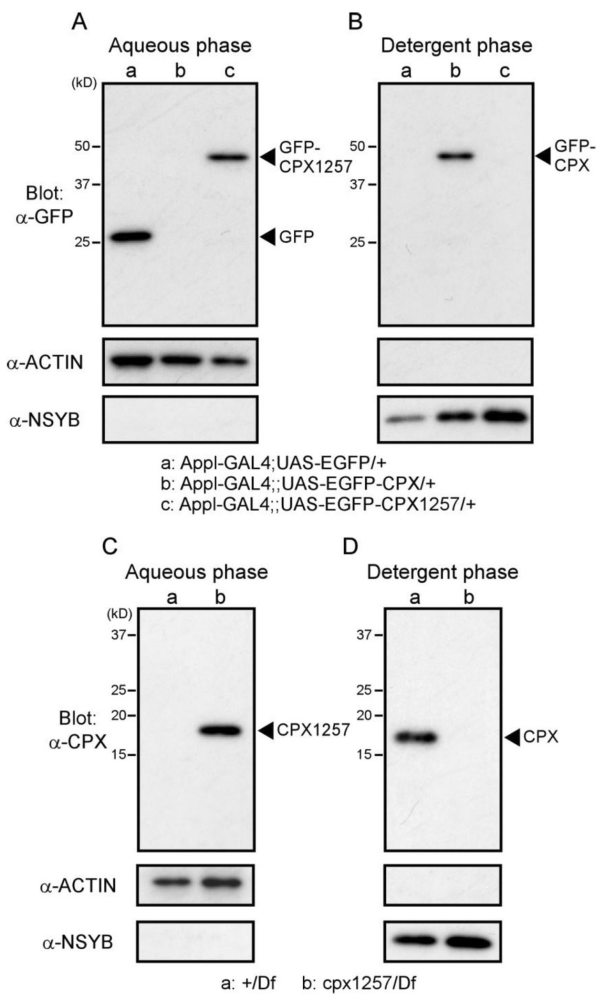

Figure 6. CPX membrane association is lost in the $\mathrm{CPX}^{1257}$ mutant which lacks the CaaX farnesylation motif

(A, B) Head homogenates from flies expressing soluble EGFP alone (a), EGFP-CPX (b) or EGFP-CPX ${ }^{1257}$ (c) in the nervous system were subjected to phase partitioning in Triton $\mathrm{X}-114$. Aqueous (A) and detergent (B) phases were then analyzed by immunoblotting with an anti-GFP antibody. While soluble EGFP partitioned into the aqueous phase as expected, EGFP-CPX was found exclusively in the detergent phase. In contrast, EGFP-CPX ${ }^{1257}$ partitioned solely into the aqueous phase. (C, D) An analysis of endogenous CPX equivalent to that in panels $\mathrm{A}$ and $\mathrm{B}$, using head homogenates from $c p_{x}$ hemizygotes (heterozygous for Df(3R)Exel6140; +/Df) (a) and cpx ${ }^{1257} / \mathrm{Df}(\mathrm{b})$. Results for endogenous CPX and CPX ${ }^{1257}$ were similar to those obtained for the corresponding EGFP fusion proteins. A control cytosolic protein, ACTIN, was detected in the aqueous phase, whereas a synaptic vesicle membrane protein, neuronal SYNAPTOBREVIN (NSYB), was detected in the detergent phase. 

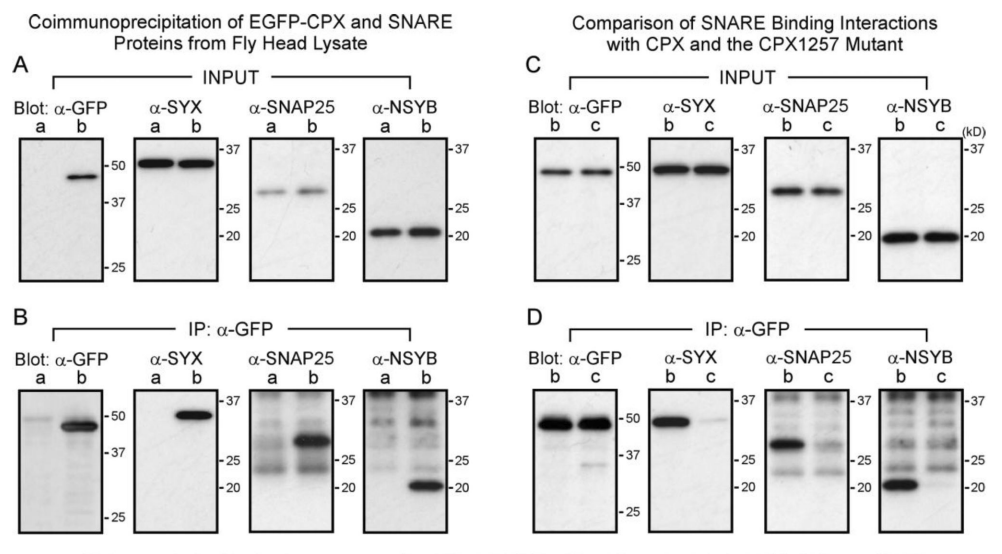

a: wild-type control lacking the transgene, b: Appl-GAL4;;UAS-EGFP-cpx/+, c: Appl-GAL4;;UAS-EGFP-cpx1257/+

Figure 7. Interaction of CPX with SNARE proteins

(A, B) Coimmunoprecipitation of EGFP-CPX and SNARE proteins from fly head lysate. An anti-GFP antibody was used to precipitate EGFP-CPX from head lysate of flies expressing EGFP-CPX in the nervous system (b) or control flies (WT) lacking the transgene to confirm specificity of the IP (a). (B) IP of EGFP-CPX and co-IP of endogenous SNARE proteins, SYNTAXIN (SYX) and neuronal SYNAPTOBREVIN (NSYB). Specific co-IP of endogenous SNARE proteins was observed only in samples expressing EGFP-CPX. Pre-IP INPUT samples are shown in Panel A. (C, D) Comparison of SNARE binding interactions with CPX and the CPX ${ }^{1257}$ mutant. Coimmunoprecipitation of EGFP-CPX and EGFP$\mathrm{CPX}^{1257}$ with SNARE proteins from fly head lysate. An anti-GFP antibody was used to precipitate EGFP-CPX or EGFP-CPX ${ }^{1257}$ from head lysate of flies expressing the respective fusion proteins in the nervous system $(b, c)$. In comparison to wild-type EGFP-CPX, co-IP of all three SNARE proteins with EGFP-CPX ${ }^{1257}$ was drastically reduced (D). However, note that, for all SNAREs, residual co-IP with EGFP-CPX ${ }^{1257}$ was detected. Pre-IP INPUT samples (C) show similar levels of EGFP-CPX and EGFP-CPX ${ }^{1257}$. 
A

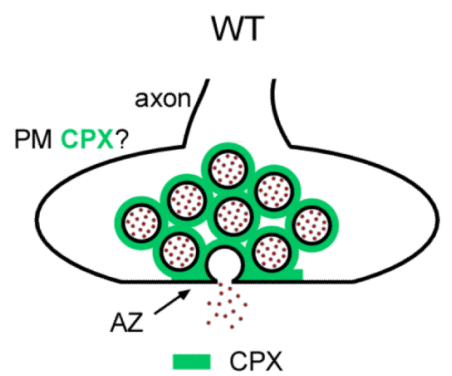

$\mathrm{AZ}$ and membrane-associated $\mathrm{CPX}$

CPX supports:

evoked fusion

clamping of spontaneous fusion
B

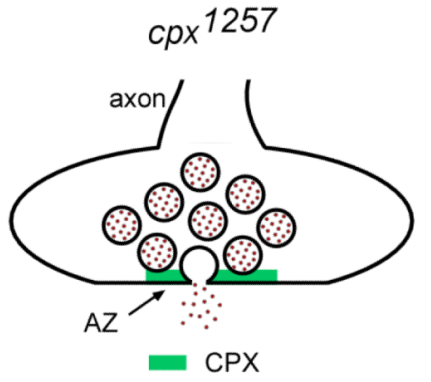

Loss of membrane-associated CPX

CPX supports:

evoked fusion

but not clamping of spontaneous fusion

Figure 8. Selective loss of CPX from presynaptic membrane compartments in the $\operatorname{cpx}^{1257}$ mutant suggests that membrane association promotes CPX function in clamping spontaneous release

(A) At wild-type synapses, CPX is associated with presynaptic membranes, including clear localization to SVs as well as the AZ where neurotransmitter release sites reside. Probable plasma membrane association of CPX (PM CPX?) is inferred from extensive farnesylationdependent CPX binding to t-SNAREs which, given retention of AZ-localized CPX in this mutant, is likely to occur in non-AZ regions of the plasma membrane. (B) In contrast, the $c p x^{1257}$ mutant exhibits an altered CPX distribution reflecting loss of broadly membraneassociated CPX but retention of CPX localization at the AZ. This altered distribution is associated with loss of CPX membrane targeting in partitioning assays, a drastic reduction in CPX-SNARE binding interactions and loss of the CPX clamping function in the $c p x^{1257}$ mutant. Notably, the activation role for CPX in evoked neurotransmitter release is retained in this mutant and this is likely to be supported by CPX-SNARE binding at the AZ. 